\title{
Objective Measures of Infant Eye-Gaze in a Single Subject: Implications for Engagement, Socialization and Communication
}

\author{
Jennifer R. Francois, Ph.D. \\ Kansas State University \\ College of Health and Human Sciences \\ 303 Justin Hall \\ 1324 Lovers Lane \\ Manhattan, KS 66506 \\ Kathy L. Coufal, CCC-SLP, Ph.D. \\ ${ }^{2}$ University of Nebraska-Omaha \\ Special Education and Communication Disorders \\ Roskens Hall 512 \\ 6001 Dodge Street \\ Omaha, NE 68182 \\ Barbara S. Chaparro, Ph.D. \\ ${ }^{3}$ Embry-Riddle Aeronautical University \\ Human Factors and Behavior Neurobiology \\ 1Aerospace Boulevard \\ Daytona Beach, FL 32114-3900
}

\begin{abstract}
Investigation of infant eye gaze behavior previously relied on subjective methods to understand infant visual preference. This study aimed to determine if differences in infant eye-gaze patterns existed between familiar and unfamiliar faces and how these patterns may relate to attachment, early engagement, and language development using an objective measure, electronic eye-tracking. To determine the existence of regularities, the eye-gaze behaviors of a 3month-old infant were recorded using the Tobii X120 eye tracker. This preliminary report details patterns observed in a single participant, as one example of the larger study, in which analyses identified similar patterns among five participants.
\end{abstract}

Keywords: eye-tracking, infants, socialization

\section{Background}

Infant acquisition of language is deeply rooted in social engagement. Early communicative acts, such as mutual gaze and nonverbal dyadic exchange, are the foundations of the complex development of human language acquisition. Environmental elements, social interactions and infant-caregiver relationships instigate and facilitate language acquisition. Previous literature, utilizing preferential looking paradigms, establisheddifferences in infant looking atfamiliar and unfamiliar faces (Field, Cohen, Garcia, \&Greenbery, 1984; Pascalis et al., 1995) and social cueing(Streri, Coulon, \&Guellai, 2012; Blehar, Liberman, \& Ainsworth, 1977). Scientistsestablished that infants show a preference for human faces (Slater \& Quinn, 2001; Slater et. al., 2010; Quinn, Tanaka, Lee, Pascalis, \& Slater, 2013) and familiar caregivers (Pascalis et al., 1995; Wagner, Luyster, Yim, Tager-Flusber\& Nelson, 2013). Earlier, investigators had to rely solely on subjective determination of looking behavior that only provided coarse descriptions of general looking patterns. Unfortunately, the accuracy of suchhistorical methods for observing young children's eye movements remained subjective. Today, fine-grained gaze analysis of specific features and characteristics of objects and humanscan now be investigated with newer methods(i.e., eye-tracking) that provide greater specificity and objectivity.This approach to understanding prelinguistic children's acquisition of language has gained popularity in recent years (Colombo, 2001; Farroni, Johnson, Brockbank, \&Simion, 2000; Hood, Willen, \& Driver, 1998). Eye tracking has also been used to examine the role of mothers' attachment behaviors on children's perception of visual cues (i.e., social cues or linguistic cues) associated with language development (Bruner, 1999; Csibra, 2010; Murray \&Yingling, 2006). Infant eye-gaze is a fundamental skill used as an early form of communication with caregivers. The connection between the infant and her caregiver facilitates this interaction. This relationship is unique and is 
conceptualized by a myriad of factors, including infant attention and recognition of familiarity. Understanding how familiarity facilitates infant social attention and engagement is an essential element of the language acquisition process. Investigations of infant eye-gaze may provide clues that point to specific and necessary face traits that differentiates a familiar caregiver from an unfamiliar one. The infant's ability to make distinctionsbetween faces of familiar/unfamiliar persons may lead to increased infant attention, social engagement, and connections to ongoing language development.

\subsection{Eye Gaze}

1.1.1 Early language acquisition. Typically, infants are born with a basicability to analyze conversational behavior, such as the capacity to initiate eye gaze in order to enter into an interaction with another person. As skills develop, infants' attempts toengage others in interactions become more intentional and sophisticated. The appearance of gazefollowing is clear by 4 months of age with increased precision as the infant ages (D'Entremont, Yazbeck, Morgan, \& MacAulay, 2007). The use of gaze-following fortifies the connection between the child and caregiver, providing an entry into a shared interest or interaction.

Didactic interactions between infants and adult caregivers during the first year of life sets in motion the development of important rules regarding social interaction. Infants' initial attempts at drawing others into social interaction begin with joint referencing. Infants also begin to understand that gaze and the information illustrated in facial expression holds meaning and constitutes a communicative intent (Bruner, 1999). As a result, they focus much of this attention on the facial area.

The dialogic "give and take" of conversation is evident between the adult and child, taking the form of mutual gaze. Like verbal dialogue, this sets the exchange paradigm and allowsthe infant to engage in a social interaction. The infant's ability to recognize their mother as a familiar caregiver and engage in mutual gaze is the result of their experience and awareness of specific facial features that promote/sustain increased infant attention. The mother helps facilitate this process by initiating interaction, sustaining engagement and using physical and auditory cues (e.g., leaning in, closer physical proximity, and using child-directed speech) to elicit her child's attention.

1.1.2 Early social development. The preference for faces and facial characteristics draw infant attention to those features that play a significant role in building the foundation for understanding social communication (Farroni et al., 2006; Farroni et al., 2002; Konishi et al., 2012; Csibra, 2010; Farroni et al., 2006; Goldstein \& Schwade, 2008; Slater \& Quinn, 2001).Senju and Csibra (2008) described how young infants depend on overt communicative signals (i.e., eye gaze, calling one's name, etc.) to engage in joint referencing. The adult caregiver's role in facilitating social and linguistic cues facilitates a connection between these elements (Goldstein \& Schwade, 2008; Legerstee, 1991b; Murray \&Yingling, 2000). Mothers who exhibit maternal sensitivity encourage their infants to engage in direct eye contact and facial cues. This promotes infant engagement in "communication" and begins the understanding of the subtleties associated with social communication and language.

Mothers, in both their communicative style and their appearance, are unique. This distinctiveness provides young infants with clues and signals about familiarity, social communication, and responsiveness. Presently, the degree to which these components impact the infants' ability to recognize familiar caregivers and how that recognition influences later language is not well understood.

\subsection{Recognition in Early Infancy}

Recognition of people during infancy has investigated how theyuse distinct facial information to determine familiarity (Bartrip, Morton, \& de Schonen, 2001). What is less well known is how familiarity facilitates attention, such that infants are more likely to show interest in attending to faces that are familiar to them. Increased interest perpetuates longer looking which in turn gives young infants a "boost" in those early cues associated with social communication. Certain adult-like capabilities regarding face processing are acquired early in a person's development (Pascalis et. al., 2011). Infant recognition of face-like paradigms and the ability to categorize human and non-human facesareall within the infant's scope a few months after birth.

Infants employ various techniques to become face experts. These include using external and internal features and automatic processes to aid in discrimination. Infants, like adults, use both categorization and recognition, as well as featural (i.e., specific characteristics) and configural (i.e., holistic) information when processing faces (Pascalis et al., 2011). A lack of consensus regarding the age at which infants switch from featural to configural processing exists. Some believe that young children rely mostly on featural processing until sometime between 4 and 7 months of age (Cashon\& Cohen, 2004), others have noted that true configural processing does not occur until approximately 10 years of age (Carey \& Diamond, 1994).Despite these inconsistencies, it seems that information gathered from featural, configural, and peripheral data play an important role in recognition. Peripherally derived visual data is an additional, integral source for the configural processing of faces. Data from the periphery would be important in the categorization 
of the face in its entirety. The role of experience in the identification of familiar faces is a recent area of increased interest (Pascalis et al., 2011). The prior review noted that experience may be integral to face processing, particularly in young infants. Experience, coupled with unique feature information, may aid infants in recognition of familiar faces. The specific facial characteristics, that infants use to assist them in determining familiarity, are not clear.

The current study seeks to understand if differences exist in eye gaze patterns when viewing familiar and unfamiliar images within a single infant participant. More specifically, this investigation was intended to address the following:

1) Do infant eye gaze patterns differ between unfamiliar and familiar face images?

2) Do infant eye gaze patterns differ within the internal and external facial features between unfamiliar and familiar face images?

\section{Methods}

\subsection{Participants}

The sample consisted of a single, 3-month-old infant who participated in a larger study investigating infant eye-gaze. The participant was born full term (i.e., 37 - 42 weeks), weighed between 5.5 and $10 \mathrm{lbs}$. at birth, and had no known neurological or visual abnormalities as determined by a maternal pregnancy and birth history that was completed by the participant's mother. The participant came from a middle-class family who resided in a mid-size Midwestern city.

\subsection{Stimulus and Apparatus}

The face stimuli pictures were black and white photo images of a familiar face (i.e., the child's mother), 5 unfamiliar faces and 5 two-dimensional, line-drawn pictures of a face, all with neutral expressions. The unfamiliar female faces were chosen to represent various ethnic groups from a convenience sample of women in the same age-range as the mothers. Each person, including the child's mother, was cloaked from the neck down with a white cloth. All photos were edited using Adobe Photoshop. Photo editing included resizing the picture, changing the coloration (i.e., making the picture black and white), and moving the image to the outer edge of the computer screen. Each infant saw a picture set combination that included a baseline and experimental picture conditions.

The eye tracking data were collected while the infant was seated in a Graco 5-in-1 highchair in front of a 17-inch computer monitor. The monitor was placed directly above the Tobii X120 eye tracker. The eye tracking system uses embedded cameras that record infrared light reflected off the cornea relative to the pupil at $120 \mathrm{hz}$. The instrument provides a high degree of accuracy, which averages within a range of 0.1 to $0.3^{\circ}$, which approximates to $0.1-0.3 \mathrm{~cm}$ area on the screen with a viewing distance of $60 \mathrm{~cm}$. When eye gaze cannot be measured from both eyes (i.e., due to head movements or head position), data from the other eye are used to determine gaze coordinates.

\subsection{Procedure}

The experimental procedure was segmented into three sessions. Upon arrival at the eye-tracking lab, the infant was placed in the highchair stationed $60 \mathrm{~cm}$ from the computer monitor. Prior to the beginning of stimulus presentation, a 2point calibration procedure was performed on the eye-tracking cameras. This involved the presentation of a moving picture accompanied by an auditory stimulus (i.e., flashing bus with a beeping sound) which was presented sequentially in two locations on the screen. This procedure was repeated if acceptable calibration was not established for both eyes in both locations.

In the baseline, familiar and unfamiliar task conditions, the infant viewed a randomized sequence of images, which included a visual attention getter, baseline image and a combination of familiar and unfamiliar images (see Figure 1). Each image within the set of three was displayed for 5 seconds per set, for a total of 15 seconds, with 5 different segment combinations shown to the infant one time.
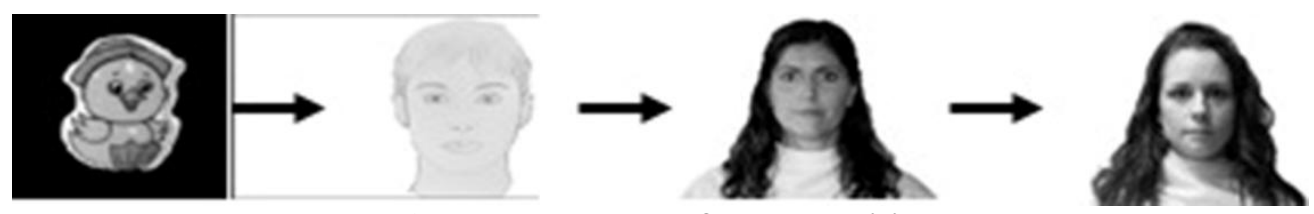

Figure 1. Example of task condition

In order to assure attention to the images on the screen, stimulus presentation was started after confirmation of onscreen looking. Continued monitoring for on-screen looking behavior occurred using a dual-monitor system and screen mirroring. Corroboration of looks was determined by viewing gaze paths on the second monitor.

\subsection{Areas of Interest (AOI)}

Each stimulus image was segmented into 9 AOIs (see Figure 2). For the current study, the AOIs identified included the following areas: 
1) left eye; 2) right eye; 3) nose; 4) mouth; 5) forehead; 6) cheeks/chin; 7) external (i.e., hair and ears); 8) neck/shoulders and; 9) area surrounding the facial image (i.e., white space surrounding the image, referred to as "off image").

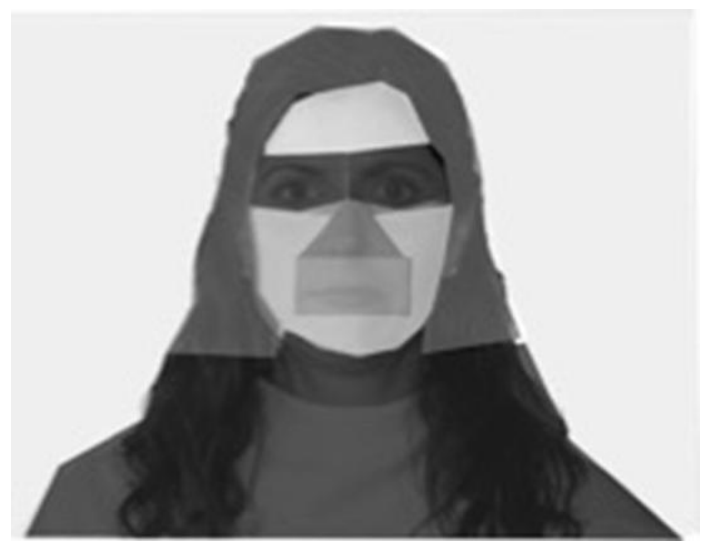

Figure 2. Example of AOI designation

\section{Data Analysis}

Eye movement data was processed using TobiiStudio ${ }^{\mathrm{TM}}$. Raw eye gaze data captured by TobiiStudio ${ }^{\mathrm{TM}}{ }_{\text {was }}$ transformed through the application of fixation filters, which used the information collected to be applied and overlaid on the visual stimuli (Tobii Technology AB, 2012). The fixation filter setting for the current study was set to the IV-T Fixation Filter. The IV-T Fixation Filter was set for a velocity threshold of $30^{\circ}$ per second. Fixations shorter than $60 \mathrm{~ms}$ were discarded. A visual analysis of the eye movement data used Microsoft Excel $^{\mathrm{TM}}$.

The current study was a single-subject design, exploring regularities in the characteristic eye-gaze patterns of one threemonth old infant. The dependent variable, fixation duration, is a metric that allows for examining what features of the face drive sustained attention, and therefore may be most salient. Fixation duration is a time measure that is calculated by summing the time spent in individual fixations within a given AOI region. Fixation duration reflects the total amount of time spent looking within an AOI. This metric is independent of the number of fixations within an AOI region, such that more fixations do not necessarily equal longer fixation time. Measures, such as fixation duration are important in understanding how young infants use gaze to search faces and persist in looking.

\section{Results}

The total proportion of fixation duration was calculated using a ratio of the total amount of time spent looking within each AOIover the total amount of time available within the experimental conditions (i.e., 50 seconds). "Off-image" looking was not included in the calculations.

Figure 3 represents the percent fixation duration for unfamiliar and familiar conditions. Small differences in the amount of time spent looking were noted between unfamiliar (i.e., 70\%) and familiar (i.e., 73\%) conditions.

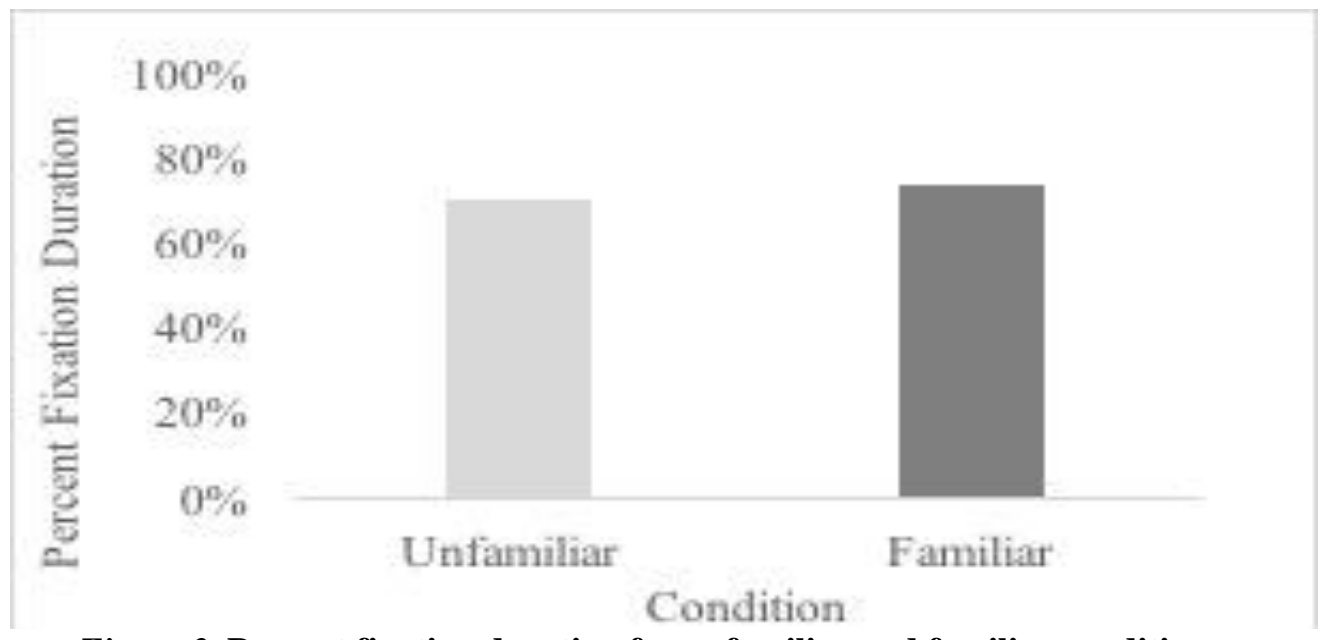

Figure 3. Percent fixation duration for unfamiliar and familiar conditions. 
Figure 4 represents the percent fixation duration for external and internal AOI regions. Differences between familiar and unfamiliarconditions were noted in the visual analysis of the data. Percent fixation duration indicated a difference in the amount of time spent looking within external and internal facial features between the familiar (i.e., familiar external (23\%); familiar internal (51\%)) and unfamiliar (i.e., unfamiliar external (16\%); unfamiliar internal (54\%)) face conditions. For both conditions more time was spent looking within the internal facial features as compared to the external facial features.

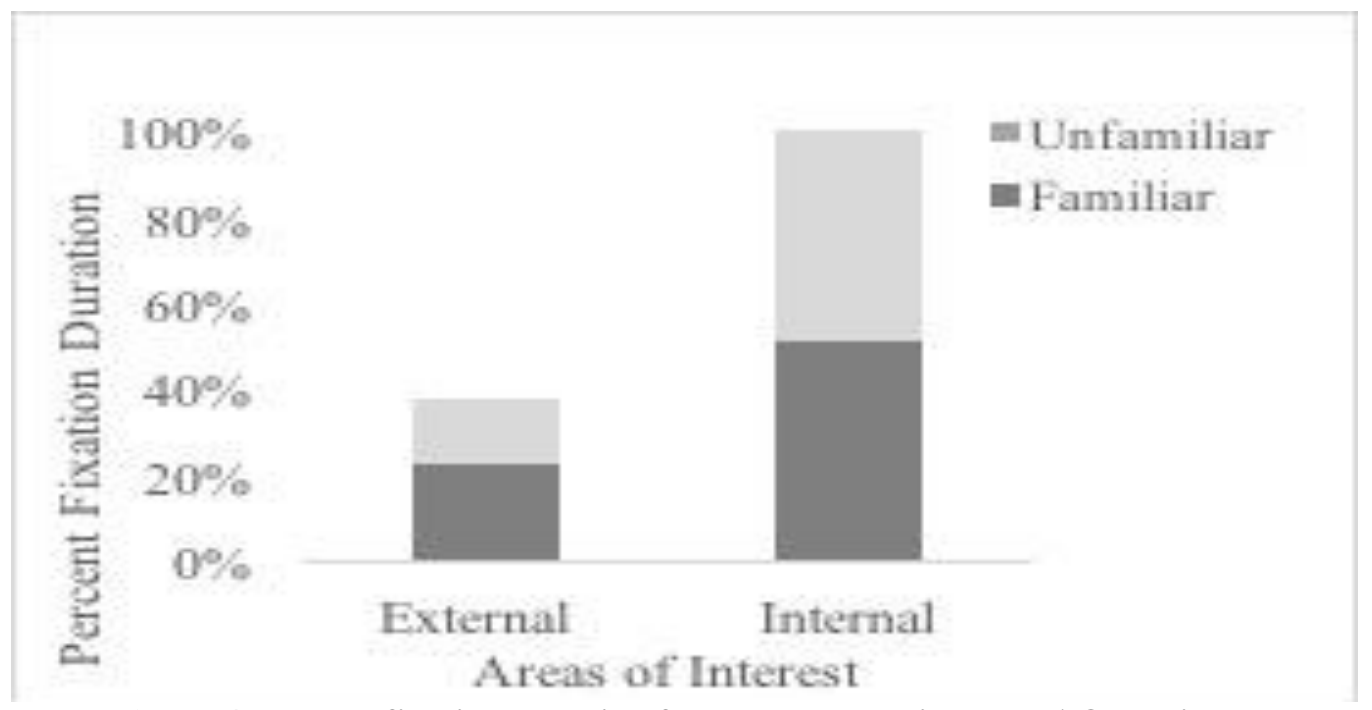

Figure 4. Percent fixation duration for external and internal AOI regions.

\section{Discussion}

The purpose of current study was to determine if the differences in eye gaze were noted between unfamiliar and familiar images for internal and external features. Comparisons in the percent fixation duration between unfamiliar and familiar conditions, as well as between targeted AOI regions of the face were conducted. Differenceswere noted between unfamiliar and familiar face conditions that contradicts previous research. Quinn and Tanaka (2009) reported young infants use internal features of the face to drive recognition for familiar faces and external features for unfamiliar faces. In contrast, these data provide evidence that internal facial characteristics were more salient than external features for both the familiar and unfamiliar face conditions. This may be related to the age of infants included in prior research (Quinn \& Tanaka, 2009) with the possibility of changes in attention shifting as the infant gets older. Previous research described changes in how infants understand the faces they see, with age-related changes occurring in the second half of the first year (Cohen \&Cashon, 2001). During the infant's first year, changes occur within the featural and configural face processing system. This includes moving from a "piece-by-piece" approach to a more holistic way of representing faces.The current study noted that internal facial features were salient for both the familiar and unfamiliar faces.It could be posited that this is suggestive of internal features being representative of the unique structure of faces in general. It is the sum of these constituent parts (i.e., internal features) that are needed to exemplify the model of the face. Without this configuration, the face would no longer look like a face. The current literature, however, is divided on whether or not very young infants have the ability to use configural face processing to drive recognition (Carey \& Diamond, 1994; Quinn \& Tanaka, 2009). The current studyprovides support for infants' configural approach to face processing as early as 3 months of age (Quinn \&Tanaka, 2009). Increased fixation duration on internal features may suggest that very young infants use them to make determinations about species-same (i.e., human vs. non-human) individuals. Recognition of familiar caregiver identity, however requires a more nuanced approach. The understanding that a face is "unique" and "specific" to one's species does not provide confirmation of identity. It is the combination of facial elements coupled with sensitive caregiving experiences that ultimately connect to familiarity. These parallel processes aid in the solicitation and sustaining of infant attention by creating an underlying social relationship.In the current study, the lack of differences between internal and external features may indicate a developmental "stage". Cues associated with recognition continue to develop as the infant ages and gains more experience with familiar caregivers. Differences over time may develop. What is less certain is whether differences existwithin the internal features that suggest young infants can distinguish between familiar and unfamiliar people. Future investigations are necessary to determine if this is the case. 
Infant engagement and attachment at these early ages attempt to use strategies like mutual gaze to interact with individuals in the infant's environment. These early communicative attempts and the ability to discriminate between familiar and unfamiliar caregivers are the building blocks oflanguage, communication, and attachment. The use of objective measures, eye-tracking, to investigate prelinguistic infant behavior provides evidence that warrants a reexamination of previously held beliefs about infant eye-gaze patterns. This, ultimately, may reveal intricacies within infant behavior and engagement that illuminate underlying linguistic processes and acquisition.

\section{Limitations}

Single subject design (SSD) has inherent limitations. Specifically geared towards small sample sizes, it allows the researcher a more detailed look within each individual participant. It does not, however, provide the liberty to make assertions across subjects and about the general population. While this does allow for a very meticulous look within an individual participant, the nature of the small sample size limits the ability to deriveconclusions and generalizes to the population. Replication across subjects is necessary to increase reliability and validity of findings. This report is illustrative of one infant participant, part of a replication study across five infants.

\section{Future Directions}

Future directions include increasing the sample size to collapse across participants in order to run statistical analysis and follow the infants longitudinally. The use of statistical analysis strengthens the reliability and validity of the data and provides ways to generalize to the population about trends and patterns in the eye gaze behaviors of young infants. Longitudinal research provides in-depth insights into incremental changes in development over time, highlighting the potential to reflect small and gradual variations in sociolinguistic development. This could lead to a greater awareness in how prelinguistic infants use familiar caregivers to facilitate their understanding of communication and social engagement.

\section{References}

Bartrip, J., Morton, J., \& de Schonen, S. (2001). Responses to mother's face in 3-week to 5-month-old infants. British Journal of Developmental Psychology, 19, 219 - 232.

Blehar, M.C., Liberman, A.F., \& Ainsworth, M. (1977). Early face-to-face interaction and its relation to later infantmother attachment. Child Development, 48, 182-194. Retrieved from: http://www.jstor.org/stable/1128897

Bruner, J. (1999). The intentionality of referring. In P. Zelazo and J.W. Astinton (Eds.), Developing theories of intention: Social understanding and self-control (pp. 329-339). Mahwah, NJ: Erlbaum.

Carey, S. \& Diamond, R. (1994). Are faces perceived as configurations more by adults than by children? Visual Cognition, 1, 253-274. doi: 10.1080/13506289408402302

Cashon, C.H. \& Cohen, L.B. (2004). Beyond u-shaped development in infants' processing of faces: An informationprocessing account. Journal of Cognition and Development, 5, 59 - 80).doi: 10.1207/s15327647jcd0501_4

Cohen, L.B. \&Cashon, C.H. (2001). Do 7-month-old infants process independent features or facial configurations? Infant and Child Development, 10, 83 - 92. doi: 10.1002/ic.250

Colombo, J. (2001). The development of visual attention in infancy. Annual Review of Psychology, 52, 337-367. doi: 0066-4308/01/0201.0337\$14.00

Csibra, G. (2010). Recognizing communicative intentions in infancy. Mind \& Language, 25 (2), 1 - 28. doi: 10.111/j.1468-0017.2009.01384.x

D'Entremont, B., Yazbeck, A., Morgan, A., \& MacAulay, S. (2007). Early gaze following and the understanding of others. In R. Flomm, K. Lee \& D. Muir (Eds.), Gaze following: It's development and significance (pp. 77 94). Mahwah, NJ: Lawrence Erlbaum Associates.

Farroni, T., Menon, E. \& Johnson, M.H. (2006). Factors influencing newborns' preference for faces with eye contact. Journal of Experimental Child Psychology, 95, 298 - 308. doi: 10.1016/j.jecp.2006.08.001

Farroni, T., Csibra, G., Simion, F., \& Johnson, M.H. (2002). Eye contact detection in humans from birth. Proceedings from the National Academy of Science, 99, 9602 - 9605. doi: 10.1073/pnas.152159999

Farroni, T., Johnson, M.H., Brockbank, M., and Simion, F. (2000). Infants' use of gaze direction to cue attention: The importance of perceived motion. Visual Cognition, 7, 705-718. doi: 10.1080/13506280050144399

Field, T. M., Cohen, D., Garcia, R., Greenbery, R. (1984). Mother-stranger face discrimination by the newborn. Infant Behavior and Development, 7, 19-25. doi: 10.1016/S0163-6383(84)80019-3

Goldstein, M.H \&Schwade, J.A.. (2008). Social feedback to infants' babbling facilitates rapid phonological learning. Psychological Science, 19, 515 - 523. 
Hood, B.M., Willen, J.D., \& Driver, J. (1998). Adults' eye trigger shifts of visual attention in human infants. Psychological Science, 9, 131-134. Retrieved from: http://www.jstor.org/stable/40063263

Konishi, Y., Okubo, K., Kato, I., Sonoko, I., Nishida, T., Takashi, K...(2012). A developmental change in the visual behavior of the face recognition in early infancy. Brain \& Development, 34, $719-722$. doi: 10.1016/j.braindev.2012.01.004

Legerstee, M. (1991b). The role of people and objects in early imitation. Journal of Experimental Child Psychology, 51, 423 - 433. doi: 0022-0965/91/\$3.00

Murray, A. D. \&Yingling, J. L. (2000). Competence in language at 24 months: Relations with attachment security and home stimulation. Journal of Genetic Psychology, 161, 133-140. Retrieved from: http://dx.doi.org/10.1080/00221320009596700

Pascalis, O., de Schonen, S., Morton, J., Deruelle, C., Fabre-Grenet, M.. (1995). Mother's face recognition in neonates: A replication and an extension. Infant Behavior and Development, 18, 79-85.

Pascalis, O., de Martin de Vivies, X., Anzures, G., Quinn, P. C., Slater, A. M., Tanaka, J. W., \& Lee, K.. (2011). Development of face processing. Wiley Interdisciplinary Reviews of Cognitive Science, 2, 666-675. doi: $10.1002 /$ wcs. 146.

Quinn, P. C., \& Tanaka, J. W. (2009). Infants' processing of featural and configural information in the upper and lower halves of the face. Infancy, 14, 474 -487. doi: 10.1080/15250000902994248

Quinn, P.C., Tanaka, J.W., Lee, K., Pascalis, Q., \& Slater, A.M. (2013). Are faces special to infants? An investigation of configural and featural processing for the upper and lower regions of houses in 3- to 7-month-olds. Visual Cognition, 21, 23-37. http://dx.doi.org/10.1080/13506285.2013.764370

Senju, A. \&Csibra, G. (2008). Gaze following in human infants depends on communicative signals. Current Biology, 18, 668 - 671. Retrieved from: http://eprints.bbk.ac.uk/2335/

Slater, A. \& Quinn, P. C. (2001). Face recognition in the newborn. Infant and Child Development, 10, 21-24. doi: $10.1002 /$ ic. 241

Slater et. al. (2010). The shaping of the face space in early infancy: Becoming a native face processor. Child Development Perspectives, 4, 205 - 211. doi:10.1111/j.1750-8606.2010.00147.x

Streri, A., Coulon, M., \&Guellai, B.. (2012). The foundations of social cognition: Studies on face/voice integration in newborn infants. International Journal of Behavioral Development, 7, 79-83. doi: 10.1177/016525412465361

Tobii Technology AB (2012). User manual Tobii Studio. Retrieved from: http://www.tobii.com/en/eye-trackingresearch/global/library/manuals/

Wagner, J.B., Luyster, R.J., Yim, J.Y., Tager-Flusberg, H., \& Nelson, C.A. (2013). The role of early visual attention in social development. International Journal of Behavioral Development, 37, 118 - 124.

doi: $10.1177 / 0165025412468064$ 